cardiaques. Nous supposons que cette efficacité thérapeutique repose à la fois sur la rapidité d'expression de la hFXN thérapeutique induite par le vecteur AAVrh10 ainsi que sur la nature hautement dynamique des mitochondries, dont le turnover est très rapide, permettant une sélection rapide des mitochondries fonctionnellement corrigées [9]. Cette possibilité de correction des cellules cardiaques est particulièrement importante dans un contexte clinique, puisque les patients ne seront pas traités en phase pré-symptomatique dans les premiers essais. Afin de transposer cette approche en clinique, plusieurs études sont en cours (1) pour déterminer l'efficacité du vecteur AAVrh10. CAG-hFXN en fonction de sa concentration (effet dose-réponse), et (2) pour optimiser l'efficacité et l'innocuité du mode d'administration chez le gros animal plus proche de la physiologie humaine. Nous continuons nos efforts de développement dans le laboratoire afin d'évaluer une approche similaire sur les symptômes neurologiques. $\diamond$

Toward gene therapy for Friedreich ataxia-associated cardiomyopathy

\section{LIENS D'INTÉR̂ิT}

H. Puccio est l'un des fondateurs scientifiques de AAVLife, une SAS dédiée à la thérapie génique pour les maladies rares.

B. Belbellaa déclare n'avoir aucun lien d'intérêt concernant les données publiées dans cet article.

\section{RÉFÉRENCES}

1. Parkinson MH, Boesch S, Nachbauer W, et al. Clinical features of Friedreich's ataxia: classical and atypical phenotypes. J Neurochem 2013; 126 (suppl 1) : 103-17.

2. Tsou AY, Paulsen EK, Lagedrost SJ, et al. Mortality in Friedreich ataxia. J Neurol Sci 2011 ; 307 : 46-9.
3. Campuzano V, Montermini L, Molto MD, et al. Friedreich's ataxia: autosomal recessive disease caused by an intronic GAA triplet repeat expansion. Science 1996 ; 271 : 1423-7.

4. Perdomini M, Hick A, Puccio H, Pook MA. Animal and cellular models of Friedreich ataxia. J Neurochem $2013 ; 126$ (suppl 1) : 65-79.

5. Perlman SL. A review of Friedreich ataxia clinical trial results. J Child Neurol 2012 ; 27 : 1217-22.

6. Wu Z, Asokan A, Samulski RJ. Adeno-associated virus serotypes: vector toolkit for human gene therapy. Mol Ther 2006 ; 14 : 316-27.

7. Perdomini M, Belbellaa B, Monassier L, et al. Prevention and reversal of severe mitochondrial cardiomyopathy by gene therapy in a mouse model of Friedreich's ataxia. Nat Med $2014 ; 20: 542-7$.

8. Puccio H, Simon D, Cossee M, et al. Mouse models for Friedreich ataxia exhibit cardiomyopathy, sensory nerve defect and Fe-S enzyme deficiency followed by intramitochondrial iron deposits. Nat Genet 2001 ; $27: 181-6$,

9. Gottlieb RA, Gustafsson AB. Mitochondrial turnover in the heart. Biochim Biophys Acta 2011 ; 1813 : 1295-301.

NOUVELLE

Production de rétines in
vitro à partir de cellules
pluripotentes humaines
Un nouvel outil thérapeutique
Sacha Reichman, José-Alain Sahel, Olivier Goureau

> La rétine, tissu sensoriel qui tapisse le fond de l'œil, est composée d'une monocouche de cellules épithéliales (épithélium pigmentaire rétinien, EPR) et de la neurorétine, qui comprend six types majeurs de neurones organisés de façon stratifiée. Cette dernière permet la conversion du signal lumineux en signal électrique par les photorécepteurs, et le message est transmis jusqu'aux cellules ganglionnaires dont les axones se regroupent pour former le nerf optique qui achemine l'information vers le cerveau. La cécité consécutive aux maladies dégénératives de la rétine, telles que les rétinopathies diabétiques ou héréditaires, la dégénérescence maculaire liée à l'âge (DMLA) et le glaucome, se caractérise principalement par une perte progressive des photorécepteurs, de l'EPR ou des cellules ganglionnaires. Si les techniques de diagnostic ont permis d'améliorer considérablement la classification de ces maladies, l'arsenal thérapeutique est toujours très insuffisant. Des approches alternatives aux traitements pharmacologiques classiques voient le jour telles que les thérapies génique, cellulaire ou prothétique, et restaurer une fonction visuelle perdue apparaît comme un des défis majeurs de la médecine actuelle. Dans ce contexte, la thérapie cellulaire constitue une des approches les plus prometteuses [1]. Les cellules souches
Institut de la vision, Inserm U968; Sorbonne Universités, UPMC-Paris 6, UMR_S968 ; CNRS, UMR 7210, 75012 Paris, France. sacha.reichman@inserm.fr j-sahel@quinze-vingts.fr olivier.goureau@inserm.fr

pluripotentes humaines, telles que les cellules souches embryonnaires ou les cellules pluripotentes induites (cellules iPS, induced pluripotent stem cells), capables de proliférer indéfiniment en culture tout en gardant leur capacité de différenciation, représentent une ressource potentiellement illimitée de cellules rétiniennes pour la transplantation [8]. Un ensemble de travaux récents rapportent la production de photorécepteurs et des cellules de l'EPR à partir de cellules iPS humaines [2, 3]. Cependant, ces méthodes apparaissent peu compatibles avec une large production de cellules d'intérêt thérapeutique. Leur faible efficacité, leur complexité, l'utilisation de composés 
exogènes et un temps de culture très long en sont les principales limites.

\section{Construction de rétines in vitro \\ à partir de cellules souches \\ pluripotentes}

Dans ce contexte, notre équipe à l'Institut de la vision, en collaboration avec une équipe d'ISTEM (Évry, France), vient de publier un protocole de différenciation en neurorétine simple, efficace et évolutif, adaptable aux normes requises pour une application de thérapie cellulaire [4]. Nous avons démontré que la culture de cellules iPS humaines à confluence dans un milieu pro-neural sans sérum est suffisante pour générer des structures de neurorétine et des cellules de l'EPR en moins de trois semaines (Figure $1 A-B)$. Ce procédé évite les étapes de formation et de sélection de corps embryonnaires (agrégats de cellules souches et de progéniteurs des trois feuillets germinaux se formant lors de l'induction de la phase initiale de la différenciation de cellules souches pluripotentes), l'addition de molécules d'induction et/ou de substrat tel que le Matrigel (un substrat de type matrice extracellulaire). Cette efficacité est
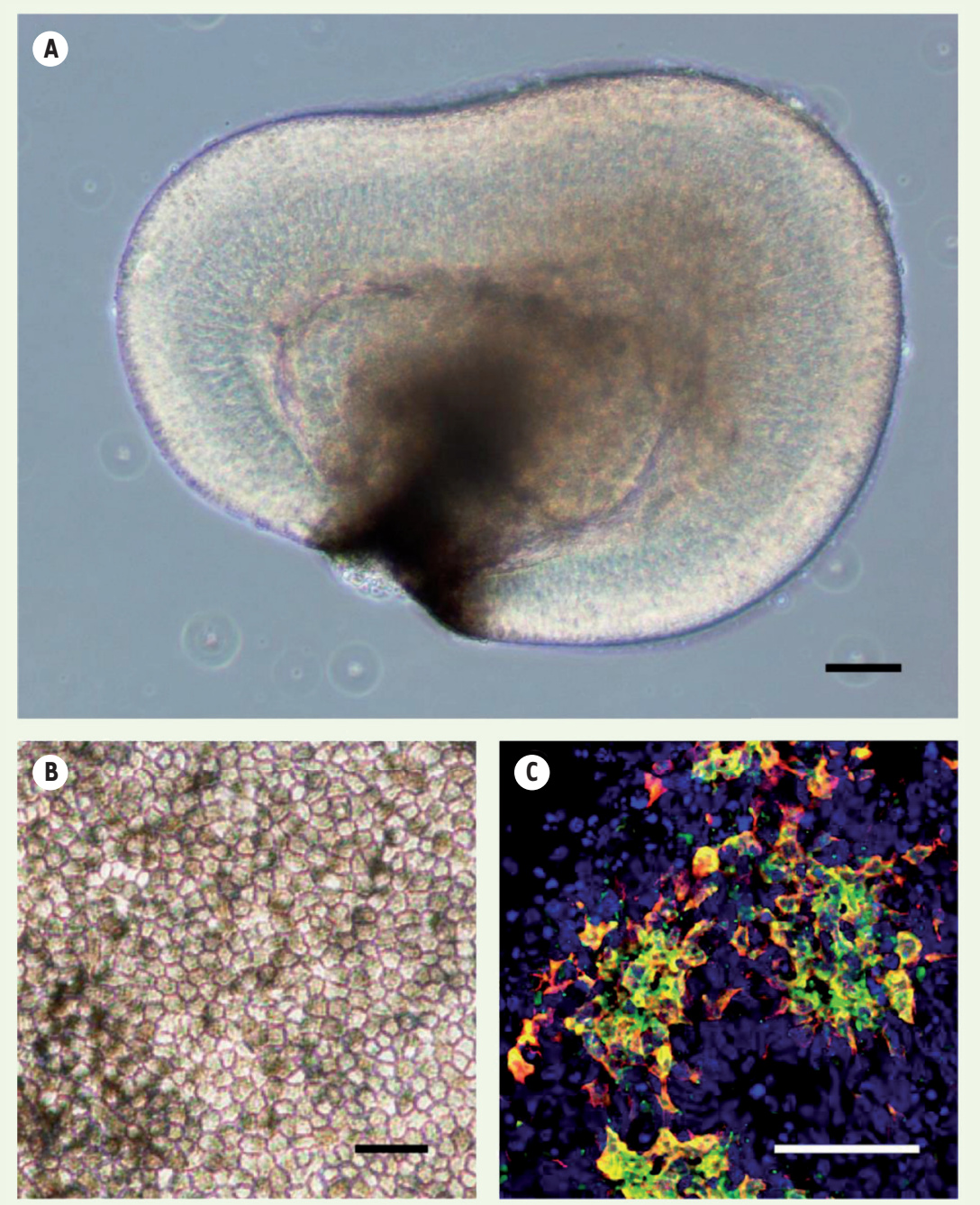

Figure 1. Génération de cellules rétiniennes à partir de cellules iPS humaines. A. Rétine in vitro en suspension après 16 jours de culture. $B$. Cellules de l'épithélium pigmentaire rétinien et C. précurseurs de photorécepteurs exprimant la Recoverine (rouge) et l'antigène de surface CD73 (vert) après 42 jours de culture. Échelle $=50 \mu \mathrm{m}$

due en partie à l'augmentation de la production endogène par les cellules iPS confluentes de DKKl (Dickkopf-1) et NOGGIN, deux inhibiteurs des voies de signalisation WNT et BMP (bone morphogenetic protein)/TGF $\beta$ (transforming growth factor). Or, l'inhibition de ces deux voies est primordiale chez l'embryon pour la différenciation du neuroectoderme et la spécification de la rétine [5]. Ces rétines in vitro obtenues au bout de 14 jours présentent un phénotype de vésicule optique similaire à ce qui est observé au cours du développement embryonnaire, ce que confirme le profil d'expression de facteurs de transcription spécifiques tels que PAX6, RAX, VSX2 (visual system homeobox 2) et MITF (microphthalmia-associated transcription factor). À partir de ce stade, la mise en culture en suspension de ces rétines in vitro permet la différenciation des progéniteurs rétiniens qu'elles contiennent en tous les types cellulaires de la rétine, d'une manière séquentielle conforme à la rétinogenèse naturelle. $\varepsilon$ n termes de cinétique, les cellules ganglionnaires, amacrines (cellules assurant des liaisons horizontales, dont les dendrites sont connectés aux cellules bipolaires et ganglionnaires) et horizontales sont détectées entre 20 et 35 jours et les précurseurs des photorécepteurs autour de 30 à 40 jours. Les photorécepteurs plus matures, exprimant les pigments visuels opsine (cônes) ou rhodopsine (bâtonnets), sont identifiés après 75 à 80 jours de culture, en même temps que les cellules gliales de Müller et les cellules bipolaires [4].

Dans la perspective d'une application en thérapie cellulaire ciblant les photorécepteurs, le stade de maturation de ces cellules est un paramètre crucial puisqu'il est établi que seuls les précurseurs des photorécepteurs sont capables de s'intégrer et de se différencier en neurones fonctionnels [1]. Dans ce contexte, nous avons mis en évidence que l'inhibition de la voie Notch, une fois les progéniteurs rétiniens engagés dans le lignage des photorécepteurs, 
augmente la proportion de précurseurs de photorécepteurs dans les rétines in vitro, en forçant les progéniteurs rétiniens à quitter précocement le cycle de division cellulaire [4]. Ces précurseurs produits à l'aide de notre protocole expriment spécifiquement l'antigène de surface CD73 (Figure 1C). La purification de cette population $C D 73^{+}$, la plus prometteuse dans une perspective de transplantation, permettra d'exclure le risque que représenterait la présence dans un mélange de cellules mal défini, de quelques cellules pluripotentes résiduelles capables de former des tératomes [6]. La capacité des précurseurs des photorécepteurs purifiés à former de nouveaux photorécepteurs fonctionnels après leur transplantation sous rétinienne constitue désormais notre prochain défi.
Rétines in vitro : source de multiples précurseurs pour une application en thérapie cellulaire

Notre protocole permet également la production rapide de nombreuses cellules ganglionnaires au sein des rétines in vitro. Si ces cellules présentent un intérêt indéniable pour le traitement du glaucome, I'un des défis majeurs et spécifiques à ce type cellulaire sera le rétablissement de leurs connexions avec le cerveau. Outre les neurones rétiniens, le procédé que nous avons mis au point permet également la génération de cellules de l'EPR (Figure IB). Ces cellules peuvent être facilement amplifiées tout en conservant leur phénotype correspondant à leur état in vivo. La création rapide de banques de cellules de l'EPR destinées au traitement futur de la DMLA et d'autres maladies liées à l'EPR est donc tout à fait envisa- geable. Notons qu'à ce jour, le seul essai clinique autorisé utilisant les cellules iPS concerne la transplantation de cellules de l'EPR chez des patients atteints de DMLA au Japon [7].

Outre son potentiel prometteur en médecine régénérative, la génération de ces différents types cellulaires rétiniens offre également l'opportunité d'étudier les mécanismes moléculaires et cellulaires impliqués dans le développement de la rétine humaine (Figure 2). Elle offre aussi de nouvelles perspectives pour avancer dans la compréhension des processus physiopathologiques qui sous-tendent les maladies dégénératives de la rétine. Ceci via la modélisation des pathologies in vitro à partir de rétines in vitro et/ ou de cellules de l'EPR issues de cellules iPS de patients porteurs de mutations (Figure 2). Ces modèles en cours de

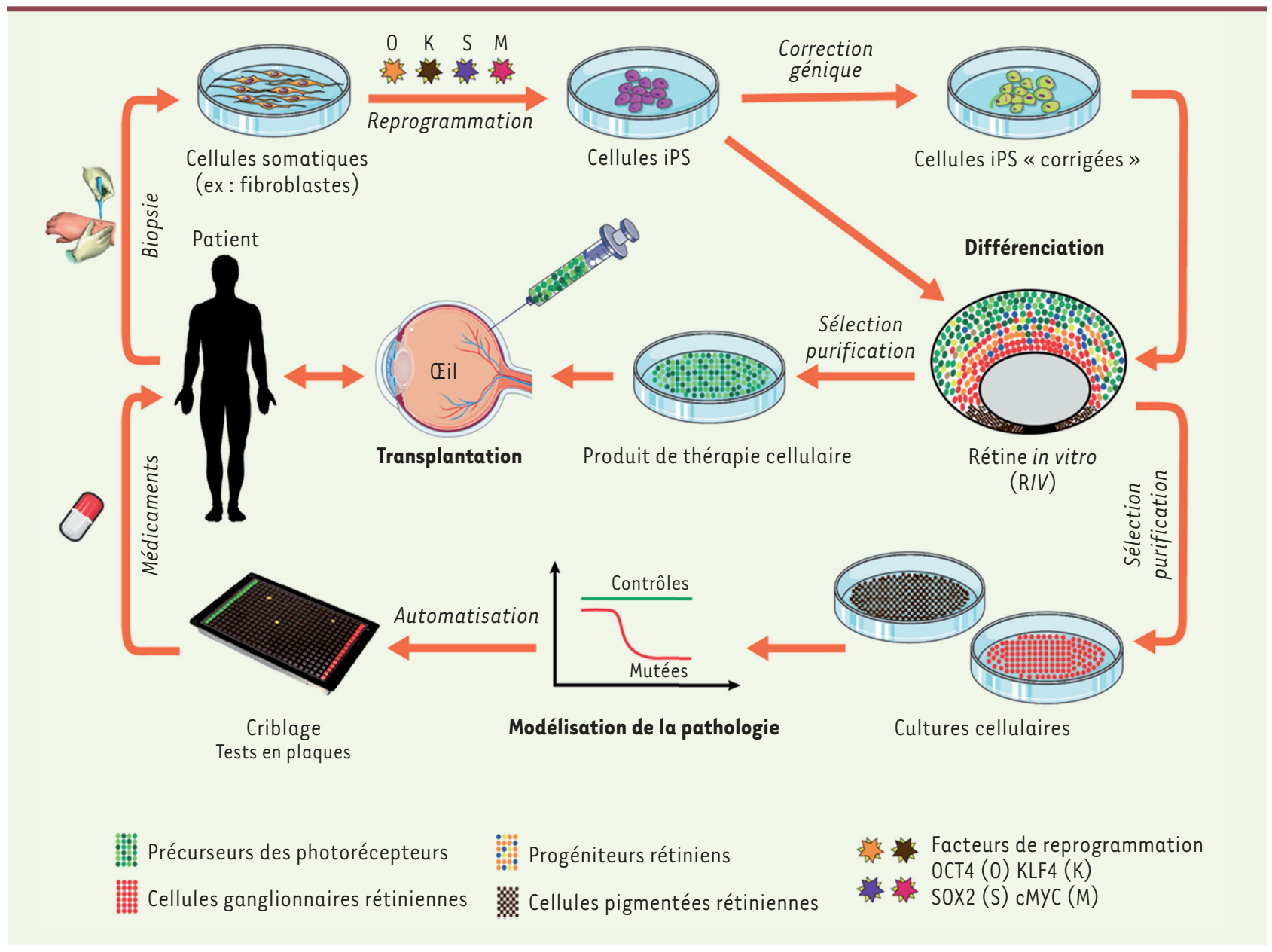

Figure 2. Schéma résumant les applications cliniques potentielles de cellules rétiniennes dérivées de cellules iPS humaines. 
développement à l'Institut de la vision pourront être utilisés pour tester l'efficacité de nombreuses molécules à visée thérapeutique par criblage à haut débit et participer ainsi au développement de nouveaux traitements pharmacologiques. $\diamond$

Production of in vitro retina

from pluripotent human stem cells: a new therapeutic tool
LIENS D'INTÉRÊT

Les auteurs déclarent n'avoir aucun lien d'intérêt concernant les données publiées dans cet article.

\section{RéFÉRENCES}

1. Pearson RA. Advances in repairing the degenerate retina by rod photoreceptor transplantation. Biotechnol Adv 2014 ; 32 : 485-91.

2. Borooah S, Phillips MJ, Bilican B, et al. Using human induced pluripotent stem cells to treat retinal disease. Prog Retin Eye Res 2013 ; 37 : 163-81.

3. Rowland TJ Buchholz DE, Clegg DO. Pluripotent human stem cells for the treatment of retinal disease. J Cell Physiol 2012 ; 227 : 457-66.
4. Reichman S, Terray A, Slembrouck A, et al. From confluent human iPS cells to self-forming neural retina and retinal pigmented epithelium. Proc Natl Acad Sci USA 2014 ; 111 : 8518-23.

5. Fuhrmann S. Eye morphogenesis and patterning of the optic vesicle. Curr Top Dev Biol 2010 ; 93 : 61-84.

6. Cui L, Guan Y, Qu Z et al. WNT signaling determines tumorigenicity and function of ESC-derived retinal progenitors. J Clin Invest 2013 ; 4 : 1647-61.

7. Bharti K, Rao M, Hull SC, et al. Developing cellular therapies for retinal degenerative diseases. Invest Ophthalmol Vis Sci 2014 ; 2 : 1191-202.

8. Peron M. La révolution 3D des cellules souches : fabrication d'une rétine in vitro. Med Sci (Paris) $2011 ; 27: 709-12$.

\section{NOUVELL}

\section{Cartographier la concentration intracellulaire d'espèces oxygénées réactives}

Cédric Bouzigues, Antigoni Alexandrou

\author{
Laboratoire optique et biosciences, CNRS UMR7645, \\ Inserm U696, École polytechnique, route de Saclay, \\ 91128 Palaiseau, France. \\ cedric.bouzigues@polytechnique.edu
}

> L'organisation dans le temps et l'espace des voies de signalisation est un élément essentiel dans le façonnage de la réponse cellulaire [1]. Ceci est vrai notamment dans les processus chimiotactiques, où l'apparition d'une organisation intracellulaire asymétrique est physiologiquement indispensable. Dans ce contexte, la signalisation par les espèces oxygénées réactives, ou ROS, est singulière. En effet, ces molécules, comme le peroxyde d'hydrogène $\mathrm{H}_{2} \mathrm{O}_{2}$, sont connues principalement pour leur activité bactéricide et les dommages induits par le stress oxydant. Elles jouent cependant en parallèle un rôle essentiel dans plusieurs voies de signalisation physiologiques [2, 3] contrôlant des réponses variées - contraction, prolifération, migration - dans de nombreux tissus (systèmes nerveux, hépatique, vasculaire, etc.). L'homéostasie locale des ROS est donc un processus vital dans la vie cellulaire pour préserver l'action physiologique sans effet nuisible [4].

\section{Comment détecter les ROS ?}

L'étude de ces questions dépend de la capacité à détecter des ROS dans des cellules vivantes de façon quantitative, dynamique et localisée. Cependant, les méthodes usuelles ne répondent pas à ces besoins. En effet, l'imagerie de ROS se fait le plus couramment par l'utilisation de la dichlorohydrofluorescéine (DCF), dont l'oxydation irréversible induit une émission de fluorescence. L'irréversibilité de ce processus et la diffusion rapide empêchent toute détection quantitative et résolue dans le temps et l'espace. La DCF permet ainsi uniquement d'identifier des voies de signalisation impliquant la production de ROS, mais pas de suivre une réponse cellulaire. Plus récemment, des protéines sensibles au peroxyde d'hydrogène, dont l'oxydation est réversible, ont été exploitées [5]. Cependant, leur gamme de détection de $\mathrm{H}_{2} \mathrm{O}_{2}$ limitée $(<500$ nM) et leur diffusion dans le cytosol rendent difficile une mesure locale et/ou quantitative. $\varepsilon$ n ce cas, comment comparer des voies de signalisation? Comment observer un profil intracellulaire de concentration de ROS ? Nous avons proposé l'utilisation de nanoparticules luminescentes à base de terres rares $\mathrm{YVO}_{4}$ : $\varepsilon u$ pour la détection de ROS dans des cellules vivantes [6] et, dans un travail récemment publié dans Chemistry and Biology, nous avons démontré son efficacité pour suivre localement le $\mathrm{H}_{2} \mathrm{O}_{2}$ intracellulaire [7]. Nous avons appliqué cette méthode aux voies de signalisation du facteur de croissance dérivé des plaquettes (PDGF), qui est impliqué dans le contrôle de la migration de cellules vasculaires [8] ou de certaines cellules tumorales [9].

Utilisation de nanoparticules à base de terres rares pour la détection de $\mathrm{H}_{2} \mathrm{O}_{2}$ Les nanoparticules de vanadate d'yttrium $\mathrm{YVO}_{4}$ peuvent être rendues luminescentes par le dopage par des ions Europium (III) qui absorbent à $466 \mathrm{~nm}$ et émettent à $617 \mathrm{~nm}$ [10]. Sous un microscope à épifluorescence équipé d'un système de détection sensible, ces particules peuvent être observées individuellement. Au contraire des fluorophores organiques ordinaires, elles ne sont pas dégradées après leur excitation. Leur illumination par une forte intensité lumineuse induit un déclin de leur luminescence, provoqué par la réduction des ions $\varepsilon u^{3+}$ en $\varepsilon u^{2+}$. Nous avons alors montré que la luminescence était restaurée par un oxydant fort, comme $\mathrm{H}_{2} \mathrm{O}_{2}$ (Figure 1). 